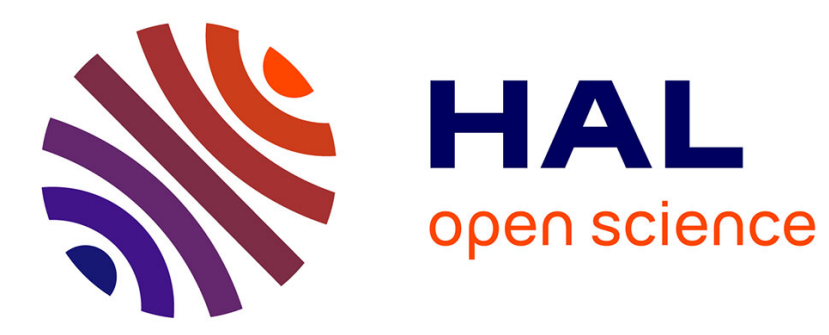

\title{
Quantum well excitonic polaritons : coupling of quantum well excitons to electromagnetic waveguide modes
}

\author{
S. Jorda
}

\section{To cite this version:}

S. Jorda. Quantum well excitonic polaritons: coupling of quantum well excitons to electromagnetic waveguide modes. Journal de Physique IV Proceedings, 1993, 03 (C5), pp.C5-59-C5-62. 10.1051/jp4:1993510 . jpa-00251596

\section{HAL Id: jpa-00251596 https://hal.science/jpa-00251596}

Submitted on 1 Jan 1993

HAL is a multi-disciplinary open access archive for the deposit and dissemination of scientific research documents, whether they are published or not. The documents may come from teaching and research institutions in France or abroad, or from public or private research centers.
L'archive ouverte pluridisciplinaire HAL, est destinée au dépôt et à la diffusion de documents scientifiques de niveau recherche, publiés ou non, émanant des établissements d'enseignement et de recherche français ou étrangers, des laboratoires publics ou privés. 


\title{
Quantum well excitonic polaritons: coupling of quantum well excitons to electromagnetic waveguide modes
}

\author{
S. JORDA
}

Institut für Theoretische Physik, Universität Regensburg, 93040 Regensburg, Germany

\begin{abstract}
The calculated dispersion curves of excitonic polaritons in GaAs quantum wells provide up to now not even a qualitative explanation for the time-of-flight experiments by Ogawa et al. [Phys. Rev. Lett. 64, 796 (1990)] which clearly show the existence of a mode with photon-like dispersion above the excitonic resonance. We show that this discrepancy can be removed by realizing that the QW constitutes a dielectric waveguide. The solution of the coupled system consisting of the exciton and the electromagnetic waveguide eigenmodes yields a drastically modified dispersion for the $T$ and $Z$ polariton as compared to previous calculations.
\end{abstract}

\section{INTRODUCTION}

The properties of QW exciton polaritons are strongly modified as compared to bulk polaritons due to the broken translational symmetry in growth direction (the $z$ direction). So far, their dispersion relations and radiative lifetimes have been derived by solving Maxwell's equations with a nonlocal excitonic susceptibility [1] as well as by diagonalizing the quantum mechanical exciton photon interaction [2]. In Refs. [1] and [2], however, the broken translational symmetry was taken into account only in the excitonic part of the coupled system, as for the free electromagnetic part the same background dielectric constant was used for well and barrier material. For the quantum field formulation this means that the vector potential was expanded in terms of creation and annihilation operators for bulk photons. As a result, for $T$ or $Z$ polarization two polariton branches were found $[1,2]$ : A lower branch (localized polariton) with infinite lifetime which starts photon-like and bends over to the exciton dispersion for large wave vectors and an upper branch (resonant polariton) which due to the coupling to the continuum of bulk photons acquires a finite lifetime. This branch starts exciton-like and ends at the line $\omega=c Q_{\|} / \sqrt{\varepsilon_{\infty}}$ where the imaginary part of the exciton self-energy diverges. These results provide no explanation for the time-of-flight experiments by Ogawa et al. [3], which clearly show the existence of a mode with photon-like dispersion above the exciton resonance. We will show that this discrepancy can be removed by realizing that the QW constitutes a dielectric waveguide, as the GaAs well has a slightly higher dielectric constant than the AlGaAs barriers. The electromagnetic eigenmodes of such a waveguide are a continuum of bulk-like radiation modes and a finite number of confined and guided modes. The radiation modes have oscillatory field components in the barriers whereas for the guided modes the field decays exponentially. The waveguide eigenmodes can in addition be classified as transverse electric (TE) or magnetic (TM) and even or odd. The latter classification refers to the transformation properties of the electric (for 
TE) or magnetic field (for TM) under the symmetry operation $z \rightarrow-z$. The transverse exciton $(T)$ couples only to even TE modes, the longitudinal exciton $(L)$ to odd TM modes (they possess an even longitudinal electric field) and the $Z$ exciton, which exists only as light hole exciton, to even TM modes. From the theory of dielectric waveguides [4] it is known that even for an arbitrarily small dielectric mismatch there is exactly one even guided TE and TM mode with zero cutoff frequency. For the $T$ polariton we will show explicitly that the coupling of the exciton to this guided mode yields a photon-like dispersion above the excitonic resonance. The modified dispersion of the $Z$ polariton will be published together with more details elsewhere. No major modifications for the $L$ polariton dispersion are expected since the cutoff frequency of the first odd guided TM mode is far above the exciton energy.

\section{INTERACTION OPERATOR}

In order to formulate the interaction between excitons and electromagnetic waveguide modes we expand as a first step the vector potential in terms of the even TE waveguide eigenmodes. For the rest of this paper we choose the conserved wave vector in the QW plane $\vec{Q}_{\|}$as being parallel to $\hat{\mathbf{e}}_{x}$. For the lowest even guided TE mode the electric field (only the $y$ component is nonvanishing) is given by [4]:

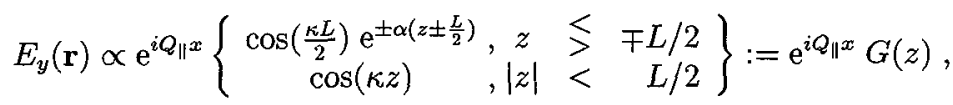

where $L$ is the QW width, $\alpha=\sqrt{Q_{\|}^{2}-\varepsilon_{\infty} \bar{\omega}_{Q_{\|}}^{2} / c^{2}}$ and $\kappa=\sqrt{\varepsilon_{\infty}^{\prime} \bar{\omega}_{Q_{\|}}^{2} / c^{2}-Q_{\|}^{2}}$. Here $\varepsilon_{\infty}\left(\varepsilon_{\infty}^{\prime}\right)$ is the dielectric constant of the barrier (well) and the dispersion relation $\tan (\kappa L / 2)=\alpha / \kappa$ has to be solved for the frequency $\bar{\omega}_{Q_{\|}}$. For the even radiative TE modes the electric field is given by

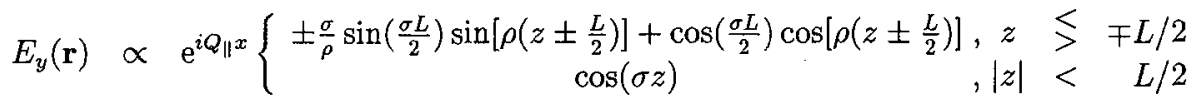

$$
\begin{aligned}
& :=\mathrm{e}^{i Q_{\|^{x}}} R(z),
\end{aligned}
$$

where $\alpha=\sqrt{\varepsilon_{\infty}^{\prime} \tilde{\omega}_{Q_{\|} \rho}^{2} / c^{2}-Q_{\|}^{2}}$ and $\rho=\sqrt{\varepsilon_{\infty} \tilde{\omega}_{Q_{\|} \rho}^{2} / c^{2}-Q_{\|}^{2}}$. The radiation modes with same $Q_{\|}$but different energy will be distinguished by the value of $\rho$.

The vector potential can now be expanded in terms of creation and annihilation operators for guided $\left(a_{Q_{\|}}^{\dagger}, a_{Q_{\|}}\right)$and radiation modes $\left(c_{Q_{\|} \rho}^{\dagger}, c_{Q_{\|} \rho}\right)$ as follows

$$
\begin{aligned}
A_{y}(\mathbf{r}) & =\sum_{Q_{\|}}\left(\frac{2 \pi \hbar c^{2} \alpha}{\varepsilon_{\infty} \bar{\omega}_{Q_{\|}} \mathcal{A}}\right)^{1 / 2}\left(a_{Q_{\|}} \mathrm{e}^{i Q_{\|} x}+a_{Q_{\|}}^{\dagger} \mathrm{e}^{-i Q_{\|} x}\right) G(z) \\
& +\sum_{Q_{\|} \rho}\left(\frac{2 \pi \hbar c^{2}}{\varepsilon_{\infty} \tilde{\omega}_{Q_{\|} \rho} \theta \mathcal{A L}}\right)^{1 / 2}\left(c_{Q_{\|} \rho} \mathrm{e}^{i Q_{\|} x}+c_{Q_{\|} \rho}^{\dagger} \mathrm{e}^{-i Q_{\|} x}\right) R(z) .
\end{aligned}
$$

$\mathcal{A}(\mathcal{L})$ is a normalization area (length) and $\theta=\frac{\sigma^{2}}{p^{2}} \sin ^{2}\left(\frac{\sigma L}{2}\right)+\cos ^{2}\left(\frac{\sigma L}{2}\right)$. Eq. (3), together with the orthogonality relations for waveguide modes [4] leads to an expression for the field energy $\frac{1}{8 \pi} \int(\mathbf{E} \cdot \mathbf{D}+$ $\left.B^{2}\right) \mathrm{d} V$ given by

$$
H_{\mathrm{em}}=\sum_{Q_{\|}} \hbar \bar{\omega}_{Q_{\|}} a_{Q_{\|}}^{\dagger} a_{Q_{\|}}+\sum_{Q_{\|} \rho} \hbar \tilde{\omega}_{Q_{\| \rho} \rho} c_{Q_{\| \rho} \rho}^{\dagger} c_{Q_{\| \rho} \rho}
$$

The exciton waveguide-mode interaction operator can be derived from the expression for the electronphoton interaction

$$
H_{\text {int }}=\frac{e}{m c} \sum_{\alpha} \int \mathrm{d}^{3} r \hat{\psi}_{\alpha}^{\dagger}(\mathbf{r}) \mathbf{p} \cdot \mathbf{A}(\mathbf{r}) \hat{\psi}_{\alpha}(\mathbf{r}) .
$$


The expansion of the field operator $\hat{\psi}_{\alpha}(\mathbf{r})$ in terms of annihilation operators for electrons in the lowest conduction- or highest valence-subband, respectively, can be taken from Ref. [2]. LH-HH mixing is accounted for by using the subband $\mathbf{k} \cdot \mathbf{p}$-method [5]. By the same procedure as outlined in Ref. [2], exciton creation $\left(B_{Q_{\|}}^{\dagger}\right)$ and annihilation operators $\left(B_{Q_{\|}}\right)$can be defined and used to write $H_{\text {int }}$ as

$$
\begin{aligned}
H_{\text {int }} & =\sum_{Q_{\|}} A_{Q_{\|}}\left(a_{Q_{\|}}+a_{-Q_{\|}}^{\dagger}\right)\left(B_{Q_{\|}}^{\dagger}+B_{-Q_{\|}}\right) \\
& +\sum_{Q_{\|} \rho} C_{Q_{\|} \rho}\left(c_{Q_{\|} \rho}+c_{-Q_{\|} \rho}^{\dagger}\right)\left(B_{Q_{\|}}^{\dagger}+B_{-Q_{\|}}\right)
\end{aligned}
$$

with the coupling constants

$$
\begin{aligned}
A_{Q_{\|}} & =\frac{e}{m c}\left(\frac{2 \pi \hbar c^{2} \alpha}{\varepsilon_{\infty} \bar{\omega}_{Q_{\|}}}\right)^{1 / 2} \Phi \frac{1}{\sqrt{3}} p_{c v} Q(\kappa) \\
C_{Q_{\| \rho} \rho} & =\frac{e}{m c}\left(\frac{2 \pi \hbar c^{2}}{\varepsilon_{\infty} \tilde{\omega}_{Q_{\|} \rho} \theta \mathcal{L}}\right)^{1 / 2} \Phi \frac{1}{\sqrt{3}} p_{c v} Q(\sigma) .
\end{aligned}
$$

$p_{c v}$ is the momentum matrix element $\left\langle c\left|p_{x}\right| x\right\rangle$ and $\Phi$ is the integral over $k_{\|}$of the product of the exciton function $\phi\left(k_{\|}\right)$with the lowest expansion coefficient of the subband $\mathbf{k} \cdot \mathbf{p}$-expansion [2]. The function $Q(x)$ is given by $Q(x)=\frac{2}{x L} \sin \left(\frac{x L}{2}\right)\left(1-(x L / 2 \pi)^{2}\right)^{-1}$.

\section{POLARITON DISPERSION}

The Hamiltonian for the coupled exciton waveguide system can now be written as

$$
H=\sum_{Q_{\|}} \hbar \Omega_{Q_{\|}} B_{Q_{\|}}^{\dagger} B_{Q_{\|}}+H_{\mathrm{em}}+H_{\mathrm{int}}
$$

where $\hbar \Omega_{Q_{\|}}$is the exciton energy which depends on $Q_{\|}$due to center-of-mass dispersion and exchange interaction [2]. Heisenberg's equations of motion for the exciton and waveguide-mode operators yield for time-periodic solutions and neglecting the antiresonant term dispersion relations of the form

$$
\hbar\left(\omega-\Omega_{Q_{\|}}\right)-\Sigma_{Q_{\|}}^{\mathrm{gui}}(\omega)-\Sigma_{Q_{\|}}^{\mathrm{rad}}(\omega)=0,
$$

with the self-energies $\Sigma_{Q_{\|}}(\omega)$ given by

$$
\Sigma_{Q_{\|}}^{\text {gui }}(\omega)=\frac{2 \bar{\omega}_{Q_{\|}}\left|A_{Q_{\|}}\right|^{2}}{\hbar\left(\omega^{2}-\bar{\omega}_{Q_{\|}}^{2}\right)}=\frac{4 \pi \mu^{2}}{3 \varepsilon_{\infty}} \frac{\omega^{2}}{\omega^{2}-\bar{\omega}_{Q_{\|}}^{2}} \alpha \Phi^{2} Q^{2}(\kappa)
$$

$\left(\mu=\frac{e \hbar}{m E_{g}}\left\langle c\left|p_{x}\right| x\right\rangle\right)$ and

$$
\Sigma_{Q_{\|}}^{\mathrm{rad}}(\omega)=\frac{1}{\hbar} \sum_{\rho} \frac{2 \tilde{\omega}_{Q_{\| \rho} \rho}\left|C_{Q_{\|} \rho}\right|^{2}}{(\omega+i \delta)^{2}-\tilde{\omega}_{Q_{\| \rho}}^{2}}
$$

with

$$
\begin{aligned}
& \operatorname{Re} \Sigma_{Q_{\|}}^{\mathrm{rad}}(\omega)=-\frac{4 \mu^{2} \omega^{2}}{3 c^{2}} \Phi^{2} \int_{0}^{\infty} \frac{\mathrm{d} \rho}{\rho^{2}+Q_{\|}^{2}-\varepsilon_{\infty} \omega^{2} / c^{2}} \frac{1}{\theta} Q^{2}(\sigma) \\
& \operatorname{Im} \Sigma_{Q_{\|}}^{\mathrm{rad}}(\omega)=\frac{2 \pi \mu^{2}}{3} \frac{\omega^{2}}{c^{2} \beta} \frac{1}{\theta} \Phi^{2} Q^{2}(\sigma),
\end{aligned}
$$

where $\beta=\sqrt{\varepsilon_{\infty} \omega^{2} / c^{2}-Q_{\|}^{2}}$. In eqs. (13) and (14) $\sigma=\sqrt{\frac{\varepsilon_{\infty}^{\prime}}{\varepsilon_{\infty}} \rho^{2}+\left(\frac{\varepsilon_{\infty}^{\prime}}{\varepsilon_{\infty}}-1\right) Q_{\|}^{2}}$ and in eq. (14) $\beta$ has to be substituted for $\rho$. 


\section{RESULTS AND DISCUSSION}

For the numerical solution of Eq. (10) we used the parameters $\mu=26.23\left(\mathrm{eV} \AA^{3}\right)^{1 / 2}, \varepsilon_{\infty}=10.2$, $\varepsilon_{\infty}^{\prime}=10.9$ characteristic for the AlGaAs/GaAs system and $\Phi=6.392 \cdot 10^{-3} \AA^{-1}$, which corresponds to an oscillator strength $f_{x y}=13 \cdot 10^{-5} \AA^{-2}$ for the LH exciton in a $50-\AA \mathrm{QW}$.

Fig. 1 shows the dispersion relation of the LH $T$-polariton. The dispersion of the guided mode and of the exciton is given by the dashed-dotted lines. As already stated the striking new feature as compared to previous calculations is the fact, that the upper polariton branch bends over to the guided mode with the corresponding photon-like dispersion. The transition from exciton-like to photon-like behaviour is very sharp. At the same time the $1 / \theta$-factor in eq. (15) cancels the singularity for $\beta \rightarrow 0$ and $\operatorname{Im} \Sigma$ remains finite, as shown in Fig. (2).

In order to compare to the experimental results by Ogawa et al. we have calculated the time delay expected from our dispersion relation for a QW with the same length as the structure described in Ref. [3]. However, our results for the time delay are by a factor of 1000 larger than those found by Ogawa et al. Also, for the width of the delay signal we find a value 10 times smaller. This difference might be due to the fact that in the experiment of Ref. [3] the QW formed the core of a much wider leaky waveguide, which may substantially modify the resulting polariton dispersion.

In conclusion, we have shown that for a correct description of the polariton structure of a single QW it is important to take into account the dielectric mismatch which is responsible for an electromagnetic confinement.
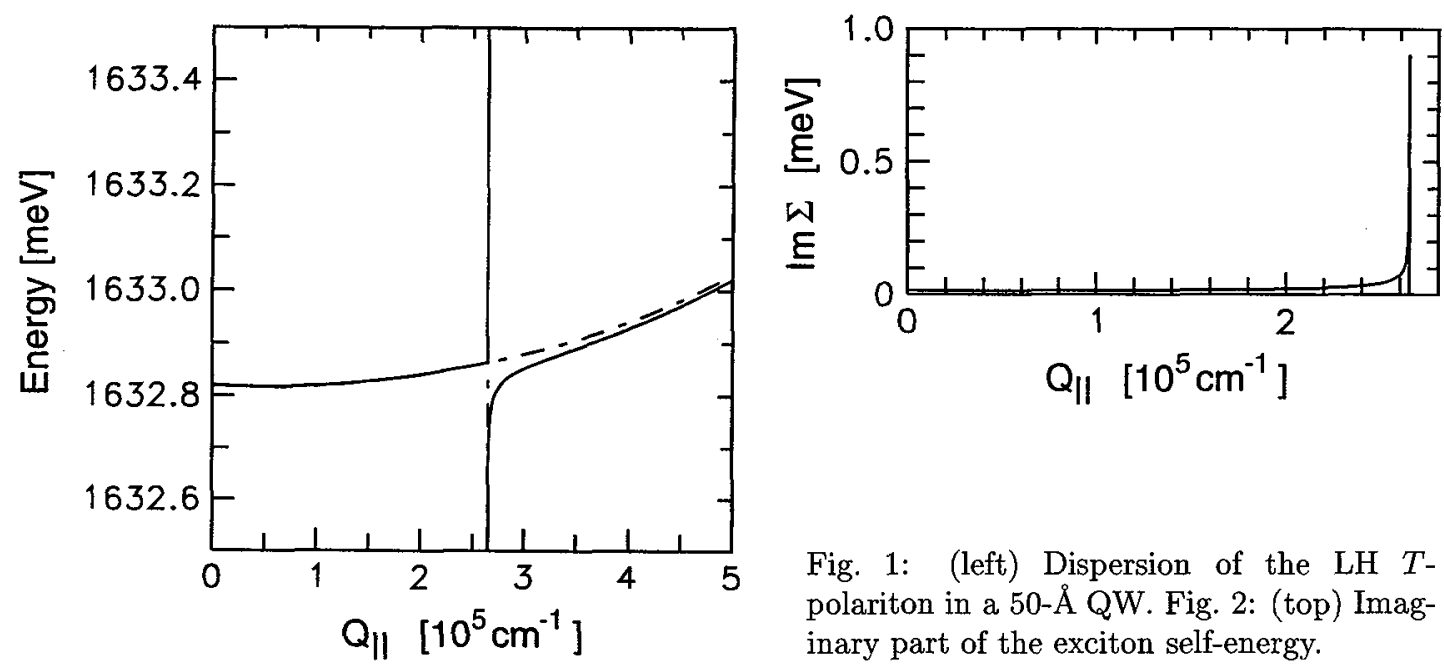

\section{References}

[1] Tassone F., Bassani F., and Andreani L.C., Nuovo Cimento D 12 (1990) 1673-1687

[2] Jorda S., Rössler U., and Broido D., Phys. Rev. B 48 (1993) 1669-1678, Jorda S., Rössler U., and Broido D., Superlattices and Microstructures 12 (1992) 85-87

[3] Ogawa K., Katsuyama T., and Nakamura H., Phys. Rev. Lett. 64 (1990) 796-799

[4] Marcuse D., Theory of Dielectric Optical Waveguides, (Academic Press, New York, 1974)

[5] Broido D., and Sham L.J., Phys. Rev. B 31 (1985) 888-892 